\title{
Modeling Longitudinal Changes in Maximal-Intensity Exercise Performance in Young Male Rowing Athletes
}

\author{
Pavle Mikulic and Tomislav Blazina \\ University of Zagreb \\ Alan M. Nevill \\ University of Wolverhampton \\ Goran Markovic \\ University of Zagreb
}

\begin{abstract}
The purpose of the current study was to examine the effect of age and body size upon maximal-intensity exercise performance in young rowing athletes. Male participants $(n=171)$ aged $12-18$ years were assessed using an "all-out" 30 -s rowing ergometer test, and reassessed after 12 months. The highest rate of performance development, which amounts to $[$ mean $(S D)]+34 \%(23 \%)$ and $+32 \%(23 \%)$ for mean and maximal power output, respectively, is observed between the ages of 12 and 13 , while this rate of development gradually declines as the athletes mature through adolescence. Performance increases with body size, and mass, stature and chronological age all proved to be significant (all $p<.05)$ explanatory variables of mean power output, with respective exponents [mean $(S E)]$ of $0.56(0.08)$, $1.84(0.30)$ and $0.07(0.01)$, and of maximal power output, with respective exponents of $0.54(0.09), 1.76(0.32)$ and $0.06(0.01)$. These findings may help coaches better understand the progression of rowing performance during adolescence.
\end{abstract}

Rowing is often referred to as a "strength-endurance" sport. A typical rowing race takes place over a 2000-m course and is, depending upon the event and upon weather conditions, characterized by 5.5-7 min of exhaustive physical effort. The correlates and prediction parameters of rowing performance have been established in numerous studies [see (10) for review], and short-term, maximal-intensity rowing ergometer performance has been shown to correlate closely with 2000-m rowing ergometer performance $(7,19,24)$. Moreover, such performance has also emerged

Mikulic, Blazina, and Markovic are with the Dept. of Kinesiology of Sport, School of Kinesiology, University of Zagreb, Zagreb, Coratia. Nevill is with the School of Sport, Performing Arts and Leisure, University of Wolverhampton, Walsall, United Kingdom. 
as an important $2000-\mathrm{m}$ rowing ergometer performance predictor $(7,19,24)$. Therefore, it appears that short-term, maximal-intensity exercise performance parameters obtained using rowing ergometry may be used for fitness assessment, as well as for talent identification and selection in the sport of rowing.

Maximal-intensity exercise refers to exercise that is performed "all-out" (27). The Wingate anaerobic test is the most widely used procedure for the assessment of such exercise, particularly in children and adolescents. Within the past several years, a modified Wingate test using rowing ergometry was developed to better reflect the coordinated bilateral upper and lower limb movements performed in rowing $(11,14,15,24)$. This rowing-specific test conducted on a Concept II rowing ergometer has been reported as reliable in terms of both intrasession reliability, with tests repeated in quick succession $(16,24)$, and intersession reliability, with tests repeated on separate days (14).

Two recent studies $(14,15)$ examined the sensitivity of the Wingate rowing test in characterizing maximal-intensity exercise performance among 12- to 18 -year-old male and female rowers grouped by age; one of these studies (15) also investigated the sex-related variation in maximal-intensity exercise performance in young rowing athletes. However, as the design of these studies was cross-sectional, any changes in the parameters of maximal-intensity exercise performance during the course of adolescence were merely inferred. Longitudinal studies, as opposed to cross-sectional studies, are particularly valuable because they may provide far greater insights into the development of physiological and performance variables in young athletes as they grow and mature. Therefore, twelve months after their initial visit, we invited participants from the study conducted by Mikulic et al. (14) to return to the laboratory and to be retested by the same researchers using the same procedures and equipment as in the original investigation. The purpose of the present longitudinal study was to apply multilevel regression modeling with multiplicative allometric equations to examine the effect of age and body size upon maximal-intensity exercise performance, as evaluated using the "all-out" 30-s rowing ergometer test, in young male rowing athletes.

\section{Methods}

\section{Participants}

In early 2010, 12-18-year old rowing athletes from nine rowing clubs, all members of the Croatian Rowing Federation, were invited to participate in a study designed to assess Wingate rowing test performance in 12- to 18 -year-old male rowers categorized by age (14). The research, conducted in the form of a longitudinal study, also aimed to investigate changes in Wingate rowing test performance in young male rowers over the course of adolescence (the objective of the current study). Two hundred and ninety-seven young rowing athletes volunteered, met the eligibility criteria (i.e., participation in rowing training for at least six months immediately before testing, attending $>75 \%$ of the total number of coach-prescribed training sessions within the same six-month period, and no reported medical problems), and submitted written informed consent forms signed by their coaches and their parents or legal guardians. The project received approval from the Ethics committee of the University of Zagreb School of Kinesiology. 
Twelve to fourteen-year-old rowers participating in this study typically train 3-5 times/week (4-7 hr/week), and importance is placed on the development of basic rowing technique. Each training session combines a rowing-specific approach, which includes on-water, ergometer, and tank rowing, with cross-training methods that mainly involve running, ball games, swimming, and body weight strength training (squats, push-ups, sit-ups, lunges, etc.). Rowers aged 15-18 years typically train 5-7 times per week (7-12 hr/week). The primary objective for this age range is the acquisition of advanced technical skills; at the same time, physical conditioning becomes more rowing-specific, with an emphasis on strength and endurance development.

One disadvantage of longitudinal studies is some inevitable amount of attrition over the course of the study. Almost one year later, all nine rowing clubs were again contacted and $171(58 \%)$ rowing athletes who had taken part in the initial assessment and were still active competitors agreed to return to the laboratory. Other participants who had been initially assessed were not available or not invited for a reassessment due to one of the following: (i) they had shifted from competitive to recreational rowing, (ii) they had forgone rowing training to participate in other sports, or (iii) they were no longer active in sports.

\section{Procedures}

Age was calculated from date of birth to the date of the examination and rounded to the nearest decimal. Stature was measured to the nearest $0.01 \mathrm{~m}$ using an anthropometer (GPM, Zurich, Switzerland), and body mass was assessed to the nearest $0.1 \mathrm{~kg}$ using a beam balance scale (Seca, Hamburg, Germany). Each participant was subsequently given 10 min to stretch and warm-up on the Concept II model $\mathrm{C}$ rowing ergometer (Morrisville, VT, USA). Concept II rowing ergometers are designed to simulate on-water rowing and are widely considered a valuable training and testing tool for rowers of all ages and ranks (10). These ergometers are air-braked, i.e., the force against which each rower must row using this type of ergometer is based on air resistance to a bladed flywheel. The flywheel is enclosed within a cover equipped with an adjustment mechanism permitting a range of possible settings from 1 to 10 .

The warm-up period consisted of continuous rowing at low to moderate intensity in accordance with the participants' usual warm-up habits, interspersed with 3 short sprints consisting of 5 maximal or near-maximal strokes. After these warm-up exercises, participants were given a two-minute rest period while the same ergometer was programmed for a 30 -s trial at the maximum damper setting (10 on the adjustment mechanism, which corresponded to a drag factor [i.e., a numerical value for the rate at which the flywheel is decelerating; this value changes in accordance with the volume of air that passes through the flywheel housing] of 156-159). The participants performed an "all-out" 30-s effort with verbal encouragement from coaches and laboratory staff members. Exercise performance was expressed as power output, as calculated and displayed by the Concept II computer and recorded by the investigators. Mean power output was the mean value for individual strokes over the 30-s trial, whereas maximal power output was the mean value for the five highest consecutive strokes during the trial. This methodology has been previously described by Riechman et al. (24), and successfully employed elsewhere $(14,15)$. 


\section{Statistical Analysis}

Descriptive statistics (mean and standard deviation) for anthropometric variables (stature and mass), chronological age and exercise performance variables (mean and maximal power output) were computed for each age group and each measurement occasion. To take full advantage of available longitudinal data, a multilevel modeling program [MlwiN (6); ] was used to investigate the effects of chronological age, stature, and mass upon mean and maximal power output during the Wingate rowing test. Multilevel analysis is an extension of ordinary multiple regression analysis when data have a hierarchical or clustered structure. In longitudinal data sets, the hierarchy may be seen as a repeated measure occasion (defined as level 1) clustered within the individuals (defined as level 2). Repeated measure concerning Wingate rowing test performance, performed as part of the current study, is an example of hierarchically structured data. Thus, when young rowing athletes are assessed on more than one occasion, two levels of variability account for a single rower's departure from their fitted growth trajectory: the measured occasion variability (level 1) and the underlying population response (level 2).

As was the case in a previous, similarly designed study in which the development of strength of quadriceps and biceps muscles in young people was modeled over a similar age range (21), a multiplicative allometric approach was adopted to describe developmental changes in the parameters of Wingate rowing test performance in young rowing athletes. This approach is characterized by all parameters being fixed with the exception of the constant (intercept term) and age parameters, which were allowed to vary randomly at level 2 (between individuals) and the multiplicative error ratio $(e)$, which varied at level 1 , thus describing the error variance within occasions. Subscripts $i$ and $j$ denote random variation at levels 1 and 2 , respectively. The "age" variable was centered about the general mean age: 15.5 years.

$$
y=\operatorname{mass}^{k 1} \cdot \text { stature }^{k 2} \cdot \exp \left(\mathrm{a}_{\mathrm{j}}+\mathrm{b}_{\mathrm{j}} \cdot \operatorname{age}+\mathrm{c} \cdot \operatorname{age}^{2}\right) \mathrm{e}_{\mathrm{ij}}
$$

This model was linearized by logarithmic transformation and multilevel regression on ln $y$ used to determine unknown parameters. Once transformed, the equation becomes:

$$
\ln y=k_{1} \cdot \ln \text { mass }+k_{2} \cdot \ln \text { stature }+a j+b j \cdot \text { age }+\mathrm{c} \cdot \operatorname{age}^{2}+\ln \left(e_{i j}\right) \text { (Eq. 2) }
$$

If we assume that the young rowers examined in the current study are geometrically similar [i.e., all their body lengths (L) are proportional to each other, all body areas are proportional to $\mathrm{L}^{2}$, and all body volumes are proportional to $\mathrm{L}^{3}$ ], then their power output should be proportional to stature ${ }^{2}$, or mass ${ }^{0.67}$ [see Jaric et al. (8)]. In that case, the appropriate sum (i.e., $3 \cdot k_{1}+k_{2}$ ) of the resulting two allometric (or scaling) exponents from eq. 2 should approximately equal 2.

\section{Results}

Age at initial assessment and physical and Wingate rowing test performance characteristics of young rowing athletes at both assessment points are presented in Table 1. The relative improvements in mean power output between the two 


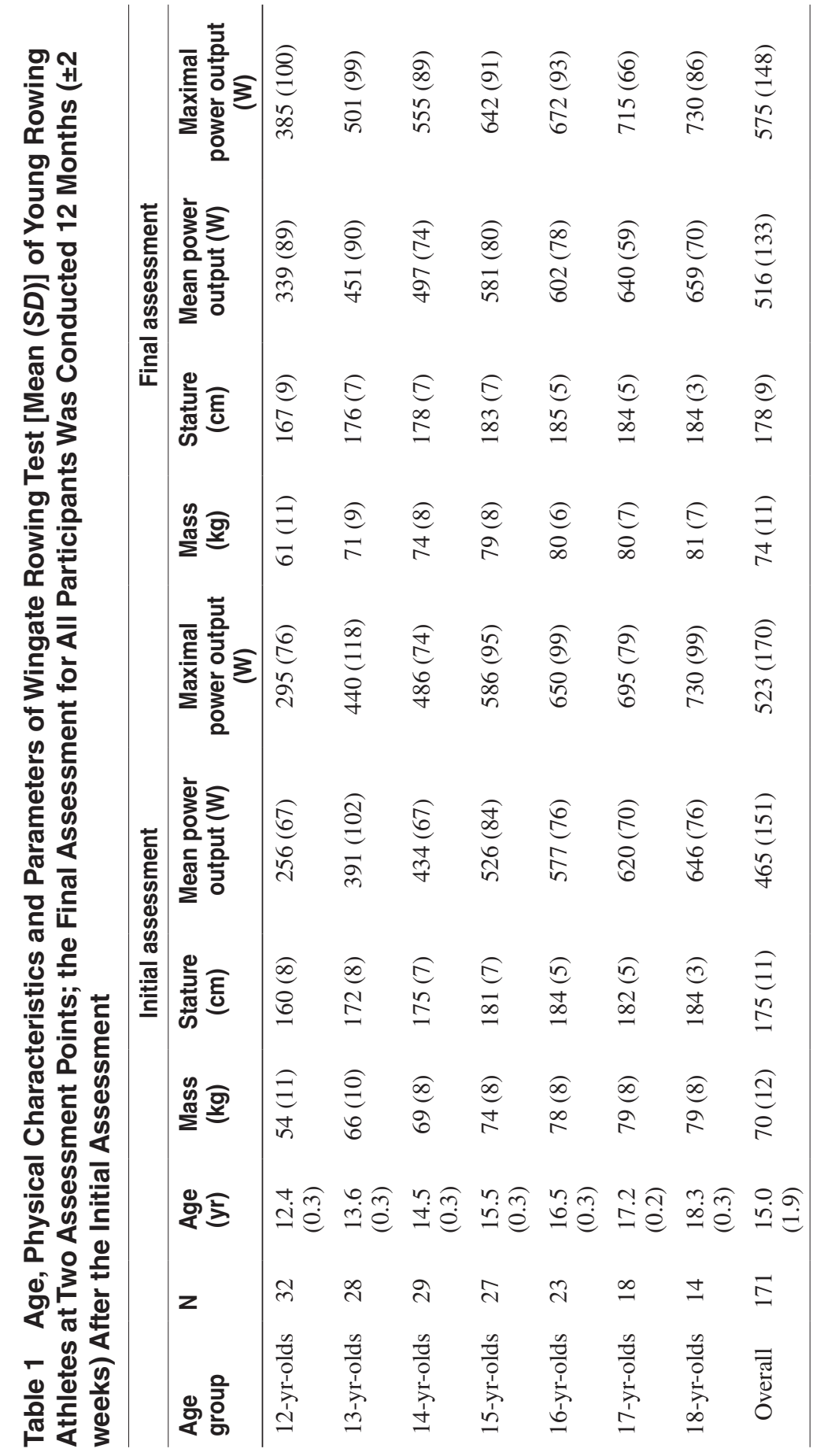


assessment occasions were [mean $(S D)]+34 \%$ (23\%) in 12-yr-olds, $+18 \%(13 \%)$ in 13 -yr-olds, $+15 \%(12 \%)$ in 14 -yr-olds, $+11 \%(8 \%)$ in 15 -yr-olds, $+5 \%(8 \%)$ in 16 -yr-olds, $+3 \%(5 \%)$ in 17 -yr-olds, and $+2 \%(3 \%)$ in 18 -yr-olds. The corresponding relative improvements in maximal power output amounted to $+32 \%(21 \%)$ in 12 -yr-olds, $+17 \%(15 \%)$ in 13 -yr-olds, $+15 \%(14 \%)$ in 14 -yr-olds, $+10 \%(9 \%)$ in 15 -yr-olds, $+4 \%(9 \%)$ in 16 -yr-olds, $+3 \%(5 \%)$ in 17 -yr-olds and $+1 \%(5 \%)$, in 18-yr-olds. Overall, between the ages of 12 and 19, mean power output increased $+157 \%$, while the corresponding increase in maximal power output amounted to $+147 \%$ (inferred from between-group differences).

The results of the multilevel regression analyses for the log-transformed indicators of Wingate rowing test performance (mean and maximal power output) are summarized in Table 2. Mass, stature and chronological age proved to be significant (all $p<.05)$ explanatory variables of mean power output with exponents [mean $(S E)]$ of $0.56(0.08), 1.84(0.30)$ and $0.07(0.01)$, respectively. The corresponding mass, stature and chronological age exponents for maximal power output were $0.54(0.09), 1.76(0.32)$ and $0.06(0.01)$, respectively.

The relationship between body size, age, and the parameters of maximalintensity exercise performance is illustrated in Figures 1,2 and 3. Mean and maximal power output clearly increase with mass (Figure 1) and stature (Figure 2). Using estimations based on elementary differential calculus (see Appendix 1 for details), we observed further acceleration in the rate of power development, which is initially greater than body size but peaks and then starts to level off at about $17-18$ years of age.

\section{Discussion}

With the possible exception of the study by Mikulic (13) in which the development of aerobic and anaerobic power in young rowing athletes between the ages of 12 and 17 was monitored, this project appears to be the first longitudinal study to report findings regarding the development of short-term, maximal-intensity exer-

Table 2 Multilevel Regression Analyses of Log-Transformed Indicators of Maximal-Intensity Exercise Performance (Mean and Maximal Power Output) of Young Rowing Athletes Adjusted for Body Size (Stature and Mass) and Age

\begin{tabular}{lcc}
\hline $\begin{array}{c}\text { Fixed Explanatory } \\
\text { Variables }\end{array}$ & $\begin{array}{c}\text { Dependent variable: Mean } \\
\text { power output } \\
\text { Value }\end{array}$ & $\begin{array}{c}\text { Dependent variable: Maximal } \\
\text { power output } \\
\text { Value }\end{array}$ \\
\hline Constant & $-5.723(1.376)$ & $-5.104(1.445)$ \\
$\ln$ (mass) & $0.557(0.085)$ & $0.540(0.091)$ \\
$\ln ($ stature) & $1.840(0.302)$ & $1.761(0.318)$ \\
Age $^{2}$ & $-0.010(0.001)$ & $-0.011(0.002)$ \\
Age (general mean) $^{\text {Age }}$ & $0.066(0.007)$ & $0.064(0.007)$ \\
\hline
\end{tabular}

All explanatory variables are significant at $\mathrm{P}<0.05$ 

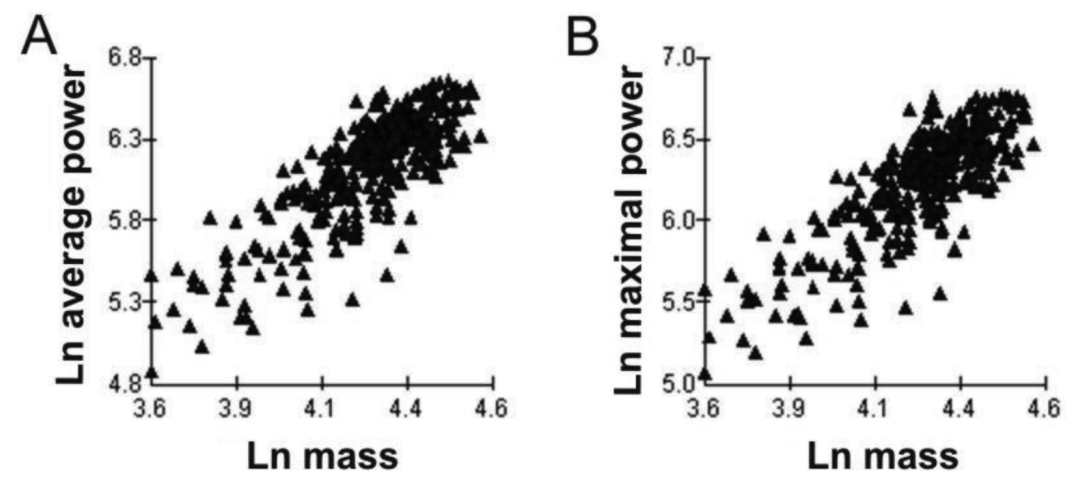

Figure 1 - Relationship between mean power output (A) and maximal power output (B) during a Wingate rowing test and body mass in young male rowing athletes. All data were log-transformed, and data from both assessment occasions $(171 \cdot 2=342$ individual measurements) were used to plot the relationship.
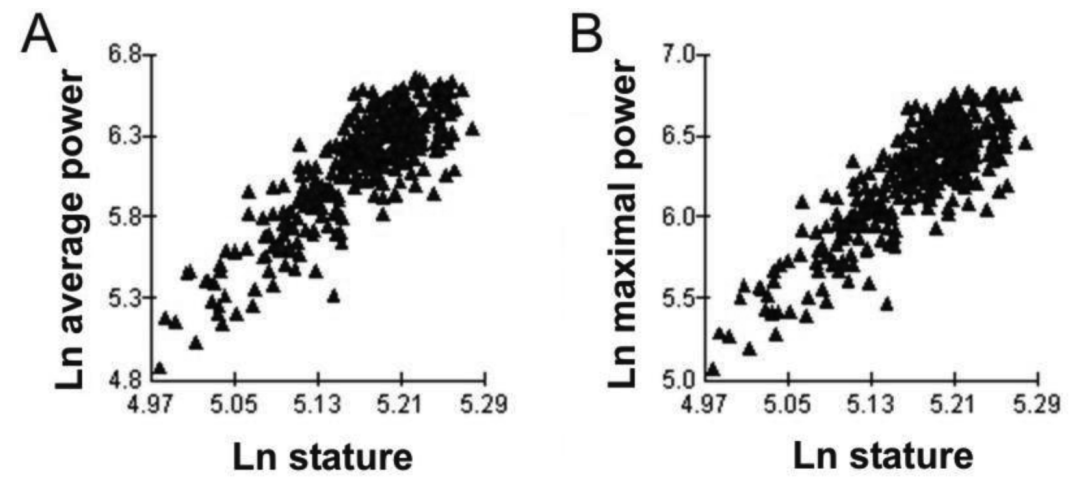

Figure 2 - Relationship between mean power output (A) and maximal power output (B) during a Wingate rowing test and stature in young male rowing athletes. All data were log-transformed and data from both assessment occasions $(171 \cdot 2=342)$ individual measurements) were used to plot the relationship.

cise performance in young rowing athletes. As we used a relatively large sample of rowing athletes aged 12-19 years on two assessment occasions, we were able to examine the development of performance in this population of athletes throughout adolescence and into very early adulthood. We summarize the main findings as follows: (i) when examined by age, the highest rate of development with regard to maximal-intensity exercise performance in young rowers is observed between the ages of 12 and 13, while this rate of development gradually declines as the athletes progress through adolescence; (ii) maximal-intensity exercise performance increases as body size grows larger in young rowing athletes, and this increase is 


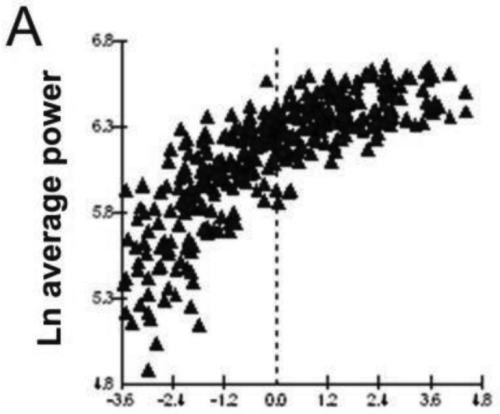

Age (yrs) from general mean

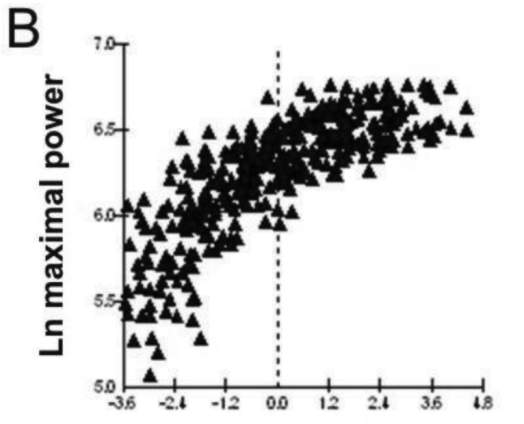

Age (yrs) from general mean

Figure 3 - Relationship between mean power output (A) and maximal power output (B) during a Wingate rowing test and chronological age in young male rowing athletes. All data were log-transformed, and data from both assessment occasions $(171 \cdot 2=342$ individual measurements) were used to plot the relationship. Note that the age is centered about the general mean: 15.5 years.

higher than that which is predicted by the theory of geometric similarity; (iii) there is an additional acceleration in the rate of maximal-intensity exercise performance development; this acceleration initially outstrips the rate of body size development but peaks and starts to level off at about 17-18 years of age.

The present longitudinal study confirmed the findings of an earlier cross-sectional report (14), reiterating that the greatest increase in maximal-intensity exercise performance in male rowing athletes is observed between the ages of 12 and 13 (see Table 1). Also highly consistent with the previous findings (14) inferred from differences observed between groups is a noticeable gradual decline in the rate of performance development as young rowing athletes age from 12 to 19 years of age. A close examination of Figure 3 suggests a higher rate of increase in performance measures between the ages of 12 and 14; afterward, this increase in performance, although still evident, becomes more gradual. Also evident from Figure 3 is a higher variability of performance measures between the ages of 12-14, as compared with performance measures after age 14 . We did not assess young rowers' levels of physical maturity on either assessment occasion; however, based on our earlier findings (16), we may carefully assume that this greater degree of variability stems in part from varying levels of physical maturity in 12- to 14-year-old rowers. Several explanations for a maturational influence on short-term, maximal intensity exercise performance have previously been proposed $(2,25)$, in particular: increases in muscle strength, muscle glycogen concentration and the rate of glycogen utilization along with maturation, a higher concentration of muscle phosphofructokinase in more mature subjects, and maturation-linked hormonal changes.

Figures 1 and 2 illustrate the dependence of short-term, maximal-intensity rowing ergometer performance upon body size. An increase in performance accompanied by an increase in both mass and stature of the participants is evident, and this observation is hardly surprising given the well-known fact that rowing performance increases with body size $(26,28)$. In the current study, the model used to analyze 
the longitudinal data set also considered individual developmental patterns with regard to performance parameters. A mathematically correct adjustment for the simultaneous increase in body size (mass and stature) and age was also taken into account. The multilevel regression analysis shown in Table 2 identified a significant increase in Wingate rowing test performance; this increase may be explained by the developmental component in body size plus an additional contribution identified by the age and age $^{2}$ components. This additional age-related contribution begins to peak at about 17-18 years of age and is likely influenced by a variety of factors. We have previously mentioned the maturation-related increases in muscle strength and muscle glycogen, as well as the presence of more efficient anaerobic metabolic pathways. In addition, as they age, rowers accumulate more experience and thus may be expected to display more technical efficiency than when they were younger, so this expectation may, in part, explain the improvement in performance levels independent of body size. Indeed, as noted by Dore et al. (5), improved motor coordination likely contributes heavily to increased power output in complex, multijoint "all-out" exercises.

Regarding the effect of body size upon Wingate rowing test performance, it should be noted that the appropriate sums of the body size scaling exponents (see Methods) for both mean and maximal power output are considerably higher than those predicted by the theory of geometric similarity $(k=3.51$ [95\% CI $=2.78-4.24]$ and 3.38 [95\% CI $=2.61-4.15]$, vs. $k=2$, respectively). It should also be noted that, if we exclude stature from the multiplicative allometric model, the resulting massscaling exponents $k$ for both mean and maximal power output increase from 0.56 to 0.88 (95\% CI $=0.75-1.00)$ and from 0.54 to 0.87 (95\% CI $=0.74-1.00)$, i.e., they appear noticeably higher than the theoretical exponent of $0.67(8,23)$. These results suggest that the maximal-intensity exercise performance, expressed as power output during the Wingate rowing test, increases along with body size in young rowers at a higher rate than predicted by the theory of geometric similarity. Several previous longitudinal studies that have modeled the effect of body size upon cycling power output in young individuals have reported similar results. For example, Armstrong and collaborators $(3,4)$ applied the same multiplicative allometric model to boys and girls as those used in the current study, reporting similar sums for the stature and mass scaling exponents for mean and peak cycling power output (3.00-3.58 vs. 3.38-3.51 obtained in the current study). Furthermore, Martin et al. (12) included body mass, lean leg volume and age in the multiplicative allometric model applied to a group of boys aged 7-17.5 years and found that cycling peak power output in boys increased with body mass at a higher rate than predicted by the theory of geometric similarity (i.e., sum of exponents for two volume-based indices of body size was $k=0.81 v s$. theoretically predicted $k=0.67$ ). The observed variation in these results could be due to interstudy variability in several factors (e.g., age, sex, maturation level, training status) that could affect the body size/performance relationship. Indeed, Jaric and colleagues (9) and Nedeljkovic and colleagues (17) demonstrated that both age and maturity level affect the relationship between body size and physical performance. Finally, a plausible explanation involving higher body size scaling exponents could be based on findings of Alexander and colleagues (1) and on the works of Nevill and colleagues $(18,20,22)$, who found that, within a variety of species including young and adult humans, larger mammals have a greater proportion of leg muscle mass in relation to their body mass to the power 
of 1.1-1.38. If the proportion of muscle mass systematically increases with, for example, mass ${ }^{1.2}$, the expected mass scaling exponents in our case should be 0.81 , i.e., close to the yielded 0.88 and 0.87 , respectively.

The fact that the experiment lacked an optimized resistance setting for each rower that would have ensured the achievement of maximal power output values may be viewed as a potential limitation of this study. A possible rebuttal is that rowing is a non-weight-bearing activity, and rowers aim to overcome the same hydrodynamic drag while in a rowing boat. Rowers also strive to overcome similar frictional and other factors to increase the angular momentum of the flywheel while rowing on an ergometer. Thus, an adjustment of the resistance setting with respect to body mass may be misleading for comparisons of different individuals' performance data. Furthermore, as indicated earlier in the text, the participants' levels of physical maturity were not assessed. The influence of physical maturity on Wingate rowing test performance has been proven in a previous study (16). It would perhaps be reasonable to assume that indicators of physical maturity, if they had been assessed, may have yielded additional information regarding the development of maximal-intensity exercise performance in rowing athletes over the course of adolescence. Unfortunately, organizational and ethical issues prevented us from collecting maturity data on this relatively large sample of young rowing athletes. Organizational issues also prevented us from collecting skinfold data on all participants during both assessments; such data would have enabled to estimate changes not only in participants' skinfold data, but also perhaps in even lean body mass and/or muscle mass. Furthermore, such explanatory variables of standard Wingate test performance $(3,12)$ would perhaps have provided valuable additional information for the current study.

In conclusion, the parameters of short-term, maximal-intensity exercise performance increase steadily in young male rowing athletes between the ages of 12 and 19. The greatest increase in performance is observed between the ages of 12 and 13 , with the rate of performance improvement decreasing gradually thereafter. As expected, power output values increase along with body size in young rowing athletes, and this increase is higher than that predicted by the theory of geometric similarity. Further acceleration in the rate of power development initially outstrips physical growth but peaks and begins to level off at about 17-18 years of age. The findings of the current study may help coaches working with young rowing athletes to gain a better understanding of the progression of rowing performance as young athletes journey through adolescence and into adulthood.

\section{References}

1. Alexander, R.M., A.S. Jayes, G.M.O. Maloiy, and E.M. Wathuta. Allometry of the leg muscles of mammals. J. Zool. 194:539-552, 1981.

2. Armstrong, N., and J.R. Welsman. Young People and Physical Activity. Oxford: University Press, 1997.

3. Armstrong, N., J.R. Welsman, and M.Y. Chia. Short term power output in relation to growth and maturation. Br. J. Sports Med. 35:118-124, 2001.

4. Armstrong, N., J.R. Welsman, C.A. Williams, and B.J. Kirby. Longitudinal changes in young people's short-term power output. Med. Sci. Sports Exerc. 32:1140-1145, 2000. 
5. Dore, E., R. Martin, S. Ratel, P. Duche, M. Bedu, and E. Van Praagh. Gender differences in peak muscle performance during growth. Int. J. Sports Med. 26:274-280, 2005.

6. Goldstein, H., J. Rasbash, and A. Plewis. A User's Guide to MLwiN. London: University of London, Institute of Education, 1998.

7. Ingham, S.A., G.P. Whyte, K. Jones, and A.M. Nevill. Determinants of 2,000 m rowing ergometer performance in elite rowers. Eur. J. Appl. Physiol. 88:243-246, 2002.

8. Jaric, S., D.M. Mirkov, and G. Markovic. Normalizing physical performance tests for body size: a proposal for standardization. J. Strength Cond. Res. 19:467-474, 2005.

9. Jaric, S., D. Ugarkovic, and M. Kukolj. Evaluation of methods for normalizing strength in elite and young athletes. J Sports Med Phys Fitness. 42:141-151, 2002.

10. Maestu, J., J. Jurimae, and T. Jurimae. Monitoring of performance and training in rowing. Sports Med. 35:597-617, 2005.

11. Mandic, S., H.A. Quinney, and G.J. Bell. Modification of the Wingate anaerobic power test for rowing: optimization of the resistance setting. Int. J. Sports Med. 25:409-414, 2004.

12. Martin, R.J., E. Dore, J. Twisk, E. van Praagh, C.A. Hautier, and M. Bedu. Longitudinal changes of maximal short-term peak power in girls and boys during growth. Med. Sci. Sports Exerc. 36:498-503, 2004.

13. Mikulic, P. Development of aerobic and anaerobic power in adolescent rowers: a 5-year follow-up study. Scand J Med Sci Sports., 2010. .

14. Mikulic, P., D. Emersic, and G. Markovic. Reliability and discriminative ability of a modified Wingate rowing test in 12- to 18-year-old rowers. J Sports Sci. 28:1409-1414, 2010.

15. Mikulic, P., and G. Markovic. Age- and gender-associated variation in maximal-intensity exercise performance in adolescent rowers. Int. J. Sports Med. 32:373-378, 2011.

16. Mikulic, P., L. Ruzic, and G. Markovic. Evaluation of specific anaerobic power in 12-14-year-old male rowers. J. Sci. Med. Sport. 12:662-666, 2009.

17. Nedeljkovic, A., D.M. Mirkov, M. Kukolj, D. Ugarkovic, and S. Jaric. Effect of maturation on the relationship between physical performance and body size. J. Strength Cond. Res. 21:245-250, 2007.

18. Nevill, A.M. Evidence of an increasing proportion of leg muscle mass to body mass in male adolescents and its implication to performance. J Sports Sci. 12:162-163, 1994.

19. Nevill, A.M., S.V. Allen, and S.A. Ingham. Modelling the determinants of $2000 \mathrm{~m}$ rowing ergometer performance: a proportional, curvilinear allometric approach. Scand J Med Sci Sports. 21:73-78, 2011.

20. Nevill, A.M., D. Brown, R. Godfrey, et al. Modeling maximum oxygen uptake of elite endurance athletes. Med. Sci. Sports Exerc. 35:488-494, 2003.

21. Nevill, A.M., R.L. Holder, A. Baxter-Jones, T.M. Round, and D.A. Jones. Modeling developmental changes in strength and aerobic power in children. J. Appl. Physiol. 84:963-970, 1998.

22. Nevill, A.M., G. Markovic, V. Vucetic, and R. Holder. Can greater muscularity in larger individuals resolve the $3 / 4$ power-low controversy when modelling maximum oxygen uptake? Ann. Hum. Biol. 31:436-445, 2004.

23. Nevill, A.M., R. Ramsbottom, and C. Williams. Scaling physiological measurements for individuals of different body size. Eur. J. Appl. Physiol. 65:110-117, 1992.

24. Riechman, S.E., R.F. Zoeller, G. Balasekaran, F.L. Goss, and R.J. Robertson. Prediction of $2000 \mathrm{~m}$ indoor rowing performance using a $30 \mathrm{~s}$ sprint and maximal oxygen uptake. J Sports Sci. 20:681-687, 2002.

25. Rowland, T.W. Developmental Exercise Physiology, 2nd ed. Champaign, IL: Human Kinetics, 2005.

26. Secher, N.H. Rowing. In: Endurance in sport, R.J. Shephard and P.O. Astrand (Eds.). Oxford: Blackwell Science, pp. 836-843. 
27. Winter, E.M., and D.P. MacLaren. Assessment of maximal intensity exercise. In: Kinanthropometry and Exercise Physiology Laboratory Manual, Volume 2: Physiology. 3rd edn. R. Eston and T. Reilly (Eds). Abingdon: Taylor and Francis, pp. 307-333.

28. Yoshiga, C.C., and M. Higuchi. Rowing performance of female and male rowers. Scand J Med Sci Sports. 13:317-321, 2003.

\section{Appendix}

Using estimations based on elementary differential calculus to model the development of mean and maximal power output during a Wingate rowing test in young rowing athletes

The model to predict power output is based on eq. 2 in the Methods. Using elementary differential calculus, we obtain: $d y / d(a g e)=b j+2 c \cdot$ age. To find the peak of the quadratic, we set dy/d(age $)=0$, i.e., $b j+2 c \cdot$ age $=0$. Since $c=-0.01$ and $\mathrm{bj}=0.066$ (mean power output; Table 2 ), and $\mathrm{c}=-0.011$ and $\mathrm{bj}=0.064$ (maximal power output; Table 2 ), solving $b j+2 c \cdot$ age $=0$ for mean power output gives $0.066+2(-0.01) \cdot$ age $=0=>$ age $=3.3$ (years). Similarly, solving $b j+2 c \cdot$ age $=0$ for maximal power output gives $0.064+2(-0.011) \cdot$ age $=0=>$ age $=2.9$ (years).

Therefore, since the age is centered about the general mean of 15.5 years, mean power output will peak at $15.5+3.3$ years $=18.8$ years, while maximal power output will peak at $15.5+2.9$ years $=18.4$ years. 